\title{
UNE NOUVELLE ONCHOCERQUE, PARASITE DE BOVIN DOMESTIQUe, AU SÉNÉGAL
}

\author{
0. BAIN*, G. VASSILIADES** et P. DELBOVE*
}

RÉSUMÉ. Une nouvelle Onchocerque, Onchocerca denkei n. sp., est trouvée dans un kyste souscutané d'un bovin domestique de race Ndama, à Kédougou. Ses affinités avec les Onchocerques des Céphalophes de la forêt gabonaise et sa rareté chez les bovins domestiques suggèrent que le réservoir de cette espèce est un Bovidé sauvage des galeries forestières de cette région.

\section{A new species of Onchocerca (Filarioidea), parasite of domestic eattle, in Senegal.}

SUMIMARY. Onchocerca denkei n. sp. was found in a subcutaneous cyst in domestic cow (race Ndama) in Kédougou, Senegal.

Its similarity to onchocercids of duikers from the Gabon forest and its rarity in domestic cattle suggest that the reservoir for this species is a wild bovid from forest galleries in the region.

Nous avons récolté en décembre 1981, à Kédougou (Sénégal), 5 nodules onchocerquiens sur des bovins Ndama, race particulière à cette région de savane soudanoguinéenne.

Quatre de ces nodules contiennent Onchocerca ochengi Bwangamoi, 1969, et le cinquième une espèce nouvelle, que nous décrivons.

\section{Matériel}

1 ㅇ mûre en plusieurs fragments (l'extrémité postérieure n'a pas été trouvée) et 1 ô : lot $229 \mathrm{NE}$, kyste no 3, M.N.H.N., Paris.

Le kyste, situé sous la peau et adhérant aux muscles du dos, a un aspect particulier : il est mal délimité, et des fragments d'onchocerques sont visibles à sa surface ; le corps de la femelle est situé dans un fourreau ; les circonvolutions du fourreau et le mâle sont enveloppés par un conjonctif très dense donnant l'apparence d'un nodule.

* Laboratoire de Zoologie-Vers, associé au CNRS, Muséum national d'Histoire naturelle, 61, rue Buffon, F 75231 Paris Cedex 05.

** I.S.R.A. Laboratoire National de l'Élevage et de la Recherche vétérinaires, B.P. 2057, Dakar, Sénégal. Accepté le 5 avril r982. 


\section{Description}

\section{- Femelle holotype (fig. 1)}

Région antérieure amincie ; œsophage divisé à mi-hauteur de l'œsophage.

Cuticule : ondulations nettes en avant de la vulve; établissement progressif de l'ornementation cuticulaire : à $8 \mathrm{~mm}$ de la tête, côtes non ondulées, stries marquées au nombre de 2-3 par intercôte ; à $12 \mathrm{~mm}$, la structure cuticulaire caractéristique est réalisée : côtes ondulées, non bifurquées ni coudées dans le plan latéral ; stries fortes, au nombre de 4 par intercôte, rarement 3 ; couche externe de la cuticule 3-4 fois plus mince que la couche interne ; cuticule non épaissie dans le plan latéral.

Fourreau épithéliomusculaire : cordes latérales à 4 files de noyaux, et aussi larges que l'ensemble des champs musculaires de la face ventrale (ou dorsale) ; musculature peu puissante, moins épaisse que les cordes latérales; dyssimétrie gauche-droite du fourreau.

Microfilaire grêle, à tête arrondie et crochet exigu.

Dimensions : longueur inconnue; le fragment antérieur, le plus long, mesure $20 \mathrm{~mm}$. Corps large de $125 \mu \mathrm{m}$ au niveau de la vulve, de $300-400 \mu \mathrm{m}$ sur le reste du corps. Cuticule épaisse de $25 \mu \mathrm{m}$; côtes espacées de 80-100 $\mu \mathrm{m}$, larges de 15-20 $\mu \mathrm{m}$. Anneau nerveux et vulve à $200 \mu \mathrm{m}$ et $620 \mu \mathrm{m}$ de l'apex ; œsophage long de $1020 \mu \mathrm{m}$, avec portion glandulaire de $680 \mu \mathrm{m}$.

Microfilaires longues de 218 à $240 \mu \mathrm{m}$ et larges de $3,5 \mu \mathrm{m}$.

\section{- Mâle allotype (fig. 2)}

Région antérieure amincie. Papilles labiales externes disposées suivant un rectangle allongé dans le plan médian et papilles céphaliques disposées suivant un rectangle allongé dans le plan latéral.

Esophage avec passage progressif de la structure musculaire à la structure glandulaire.

Ornementation cuticulaire : stries visibles au niveau de l'œsophage, encore plus accentuées sur le reste du corps. Papilles caudales disposées suivant la figure $2 \mathrm{~F}$. Spicule droit à spatule longue et fort crochet récurrent.

Dimensions : Corps long de $78 \mathrm{~mm}$ et large de $225 \mu \mathrm{m}$; stries cuticulaires espacées de $10 \mu \mathrm{m}$; anneau nerveux à $220 \mu \mathrm{m}$ de l'apex; œsophage long de $1200 \mu \mathrm{m}$; queue et spicule droit longs de $125 \mu \mathrm{m}$ et $110 \mu \mathrm{m}$; spicule gauche long de $357 \mu \mathrm{m}$, avec manche de $140 \mu \mathrm{m}$.

\section{Discussion}

Les éléments morphologiques les plus caractéristiques de cette onchocerque sont les suivants : cuticule de la femelle avec côtes ondulées, saillantes, et avec 4 stries par intercôtes, mâle de grande taille, œsophage de taille moyenne (longueur comprise 

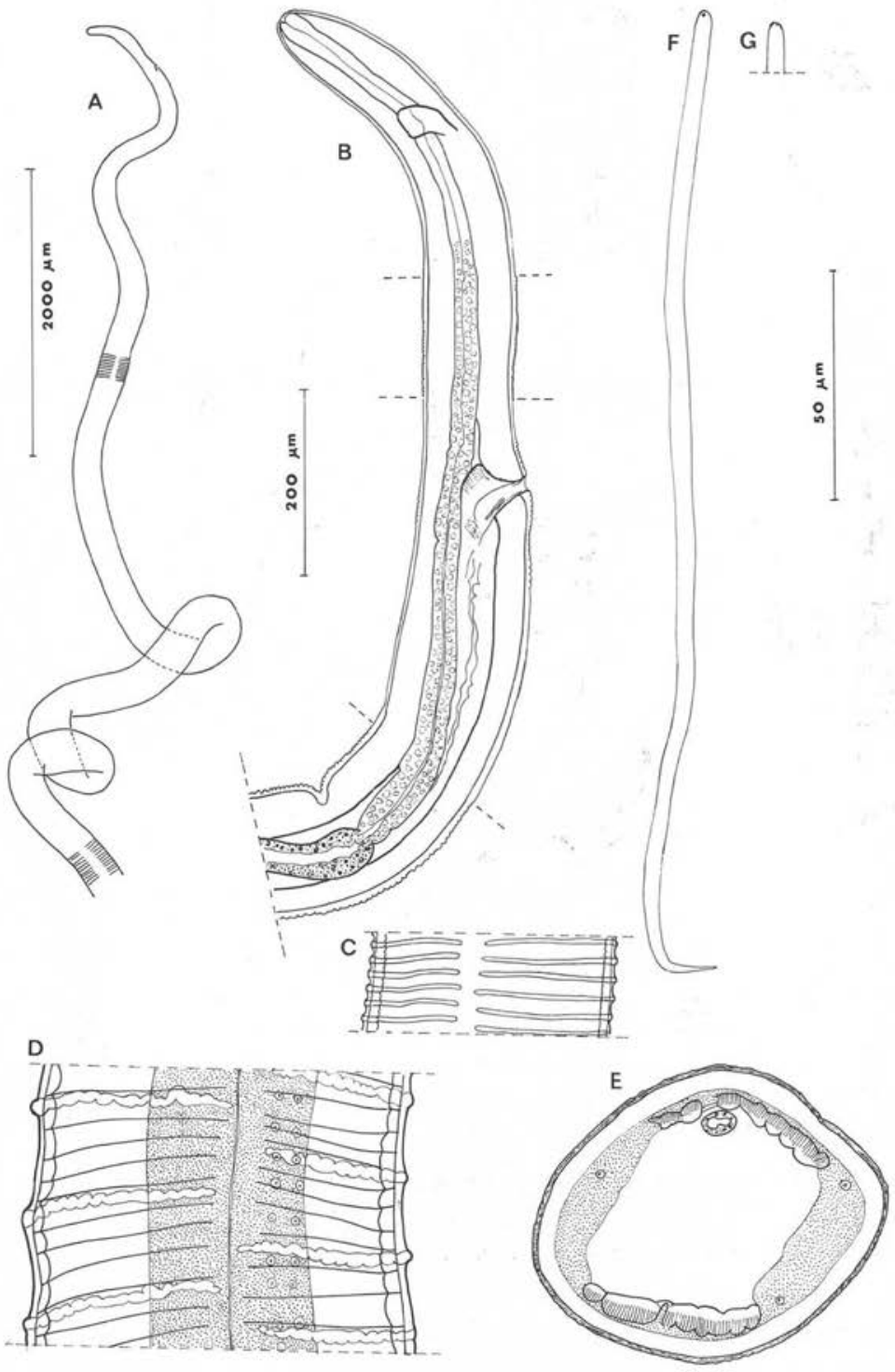

FIG. I. - Onchocerca denkei n. sp., ㅇ․ A : silhouette de la région antérieure ; B : région antérieure, vue latérale ; $\mathrm{C}$ et $\mathrm{D}$ : ornementation cuticulaire à $8 \mathrm{~mm}$ et $\mathrm{I} 2 \mathrm{~mm}$ de l'extrémité antérieure, vue latérale ; E : coupe transversale dans la région moyenne du corps ; F : microfilaire utérine, crochet vu de face; $G$ : extrémité céphalique d'une microfilaire, crochet vu de profil (éch. : A $2000 \mu \mathrm{m}-\mathrm{B}, \mathrm{C}, \mathrm{D}, \mathrm{E} 200 \mu \mathrm{m}-\mathrm{F}, \mathrm{G} 50 \mu \mathrm{m})$. 


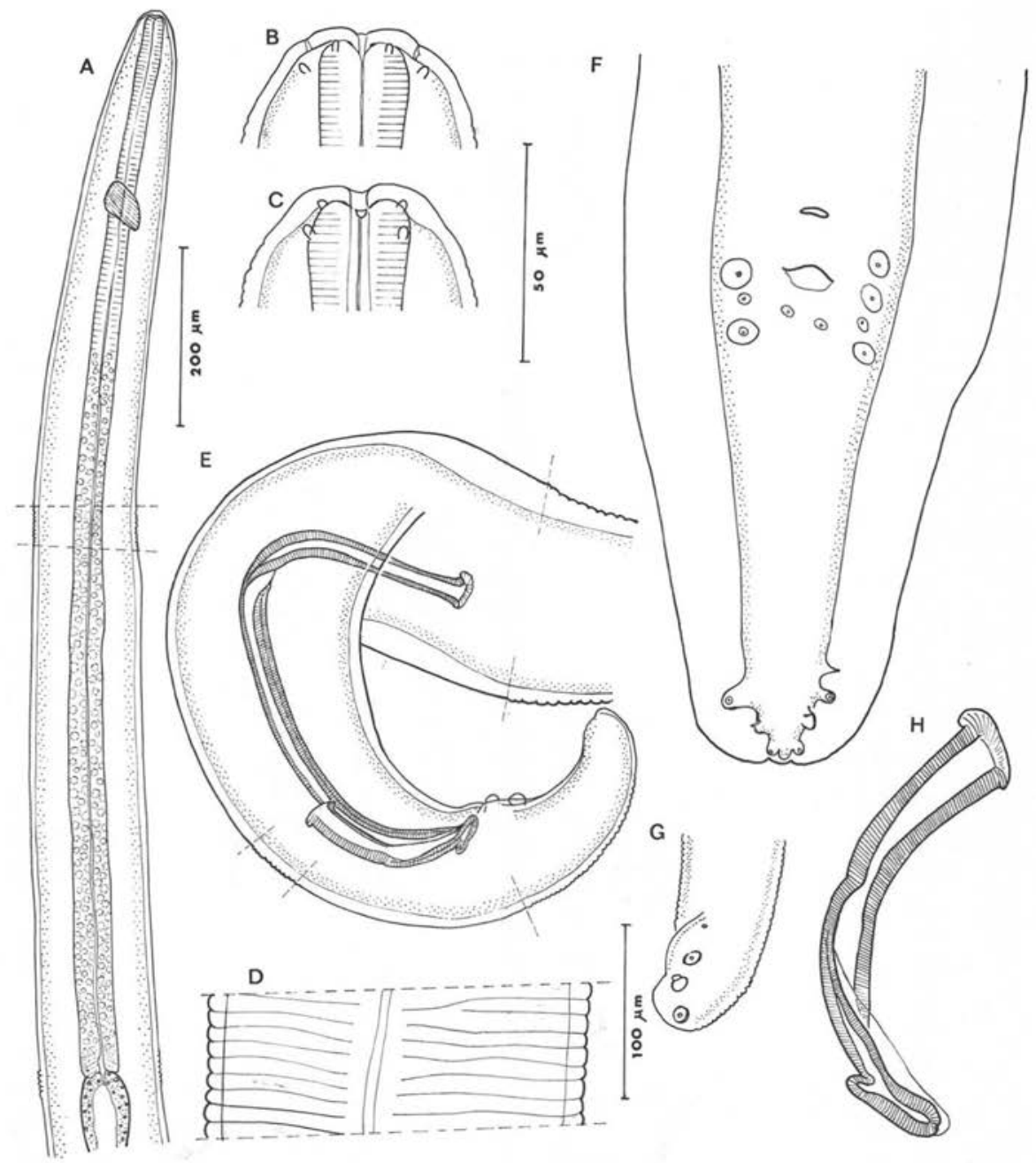

Fig. 2. - Onchocerca denkei n. sp., ô. A : région antérieure, vue latérale; B et C : tête, vues médiane et latérale ; D : ornementation cuticulaire à mi-corps, vue latérale ; $\mathrm{E}$ : région caudale ; F : queue, vue ventrale; G : extrémité caudale, vue latérale gauche ; H : spicule droit, vue latérale (éch. : A $200 \mu \mathrm{m}-\mathrm{B}, \mathrm{C}, \mathrm{D}, \mathrm{F}, \mathrm{G}, \mathrm{H} 50 \mu \mathrm{m}-\mathrm{E}$ 100 $\mu \mathrm{m}$ ).

entre 1 et $2 \mathrm{~mm}$ ), à portion glandulaire bien différenciée, vulve peu éloignée de l'anneau nerveux, groupe terminal des papilles caudales du mâle presque complet ( 7 papilles, plus les phasmides). L'espèce est donc morphologiquement proche d'O. fasciata.

Nous avons supposé qu'il pouvait s'agir de l'onchocerque du dromadaire, qui prendrait quelques caractères particuliers en s'égarant chez un bovin domestique (raccourcissement de l'œsophage, modification de la spatule du spicule droit, migration d'une paire de papilles postcloacales vers la ligne ventrale, etc.). 
De légères différences morphologiques paraissent en effet s'effectuer quand l'hôte ou la localisation de l'onchocerque sont atypiques (Ottley et Moorhouse, 1982).

Cette première hypothèse n'a pas été retenue, d'une part, parce que les différences morphologiques entre les 2 espèces sont trop importantes (la microfilaire, en particulier, est plus courte : 218-230 $\mu \mathrm{m}$, au lieu de $235-260 \mu \mathrm{m})$, et, d'autre part, parce que les aires de répartitions géographiques des dromadaires et des bovins Ndama ne se recouvrent pas et sont séparées par une distance d'au moins $100 \mathrm{~km}$.

Le deuxième groupe, qui a des affinités morphologiques avec cette espèce (cf. clé dichotomique des onchocerques, Bain, 1981), est celui des 3 espèces connues chez les Céphalophes de la forêt gabonaise : O. sonini Bain et coll., 1978, O. lerouxi Bain et coll., 1978 et $O$. beaveri Bain et coll., 1978.

L'onchocerque de Kédougou diffère cependant de ces 3 espèces par les côtes bien saillantes et ondulées chez la femelle, et par le mâle de grande taille et à grand spicule gauche $(375 \mu \mathrm{m})$, ainsi que par d'autres caractères propres à chaque espèce :

- O. sonini a une musculature puissante et une cuticule épaissie latéralement chez la femelle, une microfilaire large $(7 \mu \mathrm{m})$, avec un capuchon cuticulaire céphalique, quelques papilles très antérieures au cloaque chez le mâle.

- O. lerouxi présente, chez la femelle, une torsion spiralée du corps par rapport à l'axe longitudinal et la microfilaire est longue de 255-290 $\mu \mathrm{m}$.

- $O$. beaveri a une région antérieure très grêle, aussi bien chez le mâle que chez la femelle, et la microfilaire est longue de 248-262 $\mu \mathrm{m}$.

Cette onchocerque de Bos taurus est donc une espèce nouvelle, que nous dédions à notre collègue, le Dr. A. M. Denké et nommons Onchocerca denkei n. sp.

\section{Conclusion}

Le fait que cette nouvelle espèce ne soit découverte que tardivement chez les bovins domestiques, qu'elle paraisse relativement rare, et, surtout, le fait qu'elle soit morpho- logiquement proche des onchocerques parasites de Bovidés de forêt et non de savane, nous incitent à penser qu'il ne s'agit pas d'une espèce propre à Bos taurus et que l'espèce sera retrouvée chez un Bovidé sauvage fréquentant les forêts-galeries de la savane humide de Kédougou; Cephalophus rufilatus est fréquent dans cette région.

\section{BIBLIOGRAPHIE}

Bain O., Chabaud A. G., Landau I. : Trois nouvelles onchocerques chez des Céphalophes du Gabon. Ann. Parasitol. Hum. Comp., 1978, s3, 403-419.

BaIN O., NASher K. : Redescription de l'onchocerque du dromadaire, O. fasciata Railliet et Henry, I910. Ann. Parasitol. Hum. Comp., 1981, 56, 401-406.

BAIN O. : Le genre Onchocerca : hypothèses sur son évolution et clé dichotomique des espèces. Ann. Parasitol. Hum. Comp., I981, s6, 503-526.

Ottley M. L., Moorhouse D. E. : Morphological variations in Onchocerca sp. from atypical hosts and sites: the validity of $O$. stilesi. Ann. Parasitol. Hum. Comp., r982 (à paraitre). 